Journal Club

Editor's Note: These short, critical reviews of recent papers in the Journal, written exclusively by graduate students or postdoctoral fellows, are intended to summarize the important findings of the paper and provide additional insight and commentary. For more information on the format and purpose of the Journal Club, please see http://www.jneurosci.org/misc/ifa_features.shtml.

\title{
Semantic Organization of Body Part Representations in the Occipitotemporal Cortex
}

\author{
(D)Arran T. Reader \\ School of Psychology and Clinical Language Sciences, University of Reading, Reading, RG6 6AL, United Kingdom \\ Review of Bracci et al.
}

Experiments using functional magnetic resonance imaging ( $\mathrm{fMRI})$ have revealed that the ventral and lateral occipitotemporal cortices (VOTC and LOTC, respectively), areas often associated with high-level visual processing (GrillSpector and Malach, 2004), show preferential activation during the observation of body parts. Early results examining preferential brain activation during observation of body parts highlighted the importance of a region in the LOTC. This was termed the extrastriate body area (EBA) by Downing et al. (2001), who found that this region responded more strongly to images of body parts than to various control stimuli. Supporting these results, Urgesi et al. (2004) applied transcranial magnetic stimulation over EBA, which resulted in a reduced ability to discriminate between body parts. More recently, Bracci et al. (2010) found an area of the LOTC that responded preferentially to the observation of hands, suggesting that body part representation in the OTC is likely to be heterogeneous.

While the organization of body part representations (i.e., somatotopy) in the somatosensory and motor cortices is well

\footnotetext{
Received 0ct. 14, 2015; revised Nov. 19, 2015; accepted Nov. 23, 2015. Many thanks to Dr. Nicholas P. Holmes for his comments on the manuscript, and for his continued supervision and support.

The author declares no competing financial interests.

Correspondence should be addressed to Arran T. Reader, School of Psychology and Clinical Language Sciences, University of Reading, Earley Gate, Whiteknights Road, Reading, RG6 6AL, UK. E-mail: a.reader@pgr.reading.ac.uk.

DOI:10.1523/JNEUROSCI.3766-15.2016

Copyright $\odot 2016$ the authors $\quad 0270-6474 / 16 / 360265-03 \$ 15.00 / 0$
}

established, little evidence existed for similar organization in the OTC until relatively recently. Orlov et al. (2010) found that distinct areas of the OTC represented different visually presented body parts. They also showed that the organization of these representations was not accounted for simply by the shape of the body parts. Such findings suggested that body part representations in the LOTC and VOTC might be organized according to one or more criteria, for example shape or function. In a recent issue of The Journal of Neuroscience, Bracci et al. (2015) further examined how body-part-related activity in the OTC is organized. Using fMRI and a technique called representational similarity analysis (RSA; Kriegeskorte et al., 2008), they tested five different models of body part organization in the OTC. Their results revealed that a model based on semantic similarity best accounted for the organization of body part representations in the OTC.

Bracci and colleagues (2015) showed participants images of whole bodies, hands, feet, arms, legs, chests, waists, upper faces, lower faces, and chairs, while they performed a one-back repetition detection task and fMRI data were collected. Regions of interest (ROIs) in the LOTC and VOTC were defined in each participant by contrasting the bloodoxygenation level dependent (BOLD) response to whole bodies versus chairs. ROIs within the OTC were found in both hemispheres for all participants. Control regions in the occipital cortex (OC) were defined by the opposite contrast: chairs versus whole bodies. Multivoxel pattern analysis, a tool for comparing patterns of activation across multiple voxels, was then used to compare the similarities of activation among the eight remaining body part activations in the LOTC and VOTC. These patterns were correlated across the different body parts to create an eight-by-eight neural dissimilarity matrix (Bracci et al., 2015, their Fig. 3A).

The authors used RSA, which involves comparing dissimilarity matrices between a model and measured brain activation (Fig. 1). Using RSA, different theoretical models can be compared to discover the degree to which each can explain the observed brain activity. To use RSA, the authors created dissimilarity matrices for five possible models of body part organization: physical shape similarity (computed using the shape context algorithm; Belongie et al., 2002), perceived shape similarity (based on six independent participants' arrangement of body parts based on shape similarity), physical proximity in the body, organization following the cortical sensory and motor homunculi, and semantic similarity (Bracci et al., 2015, their Fig. 2A). These dissimilarity matrices were then compared statistically with the neural dissimilarity matrices of each of the four ROIs (left and right LOTC and VOTC).

In the primary analyses, the authors replicated the body-part-specific activation of OTC reported previously. Moreover, they found that only the perceived 
shape, physical proximity, and semantic similarity models were significantly and positively related to neural similarity in the LOTC and VOTC. The physical shape and cortical homunculus models failed to account for any additional variance in the neural similarity. When these models were directly compared, however, the semantic similarity model was significantly more related to neural similarity than any of the other models, suggesting that the organization of body part representations in the LOTC and VOTC is at least partly semantic. In examining the neural dissimilarity matrices, the authors found three distinctly organized clusters: effectors (hands, feet, legs, arms), noneffectors (chests, waists), and faces (upper faces, lower faces). To check if nearby regions showed complementary organization, the analysis was performed again on the left and right OC (control ROIs). Perhaps unsurprisingly, considering the importance of the occipital cortex in processing lower-level visual stimuli, only the shape similarity models were significantly related to patterns of BOLD response in these areas.

Bracci et al. (2015) provide evidence that the organization of body part representations in the OTC is at least partially semantic, though such a model alone could not fully explain the neural similarity. As the authors note, this may have been due to "imperfections in the models and/or the existence of additional organizational principles that we did not consider" (p. 12983). The former may be most likely, considering that the method by which the semantic similarity model was defined (frequency of body part word co-occurrence in written text) may not adequately reflect the way in which such semantic similarity is represented in the OTC. It may be the case that semantic organization in the OTC is quite different than that which can be found in the distribution of words in text. For example, while the combination of body parts in text may reflect a wide variety of possible functions (i.e., both feet and hands being used in climbing, or both hand and mouth being used in eating), their representation in the OTC may be related to more specific general functions such as expressing emotion, or moving aversive/ attractive stimuli away from/towards the body. This may also explain why the physical proximity model was also positively associated with neural similarity because, for example, hands are almost always used in combination with the arms.
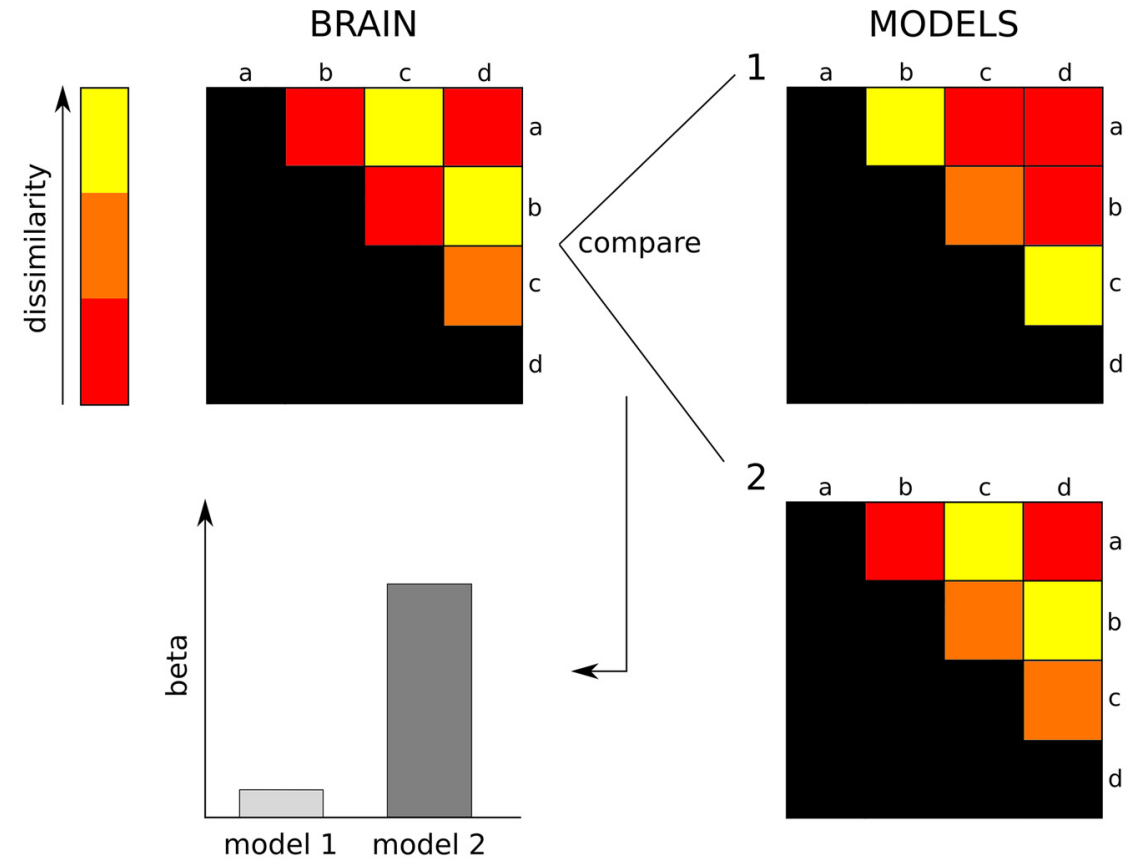

Figure 1. Simplified overview of RSA as used by Bracci et al. (2015). Correlation-based multivoxel pattern analysis can be used to determine voxelwise dissimilarity between neural activity related to the stimuli a $-\mathrm{d}$. This is used to create a neural dissimilarity matrix. A dissimilarity matrix is a square, symmetrical matrix that compares the similarity between pairs of elements. Diagonal pairs (showing the dissimilarity between an element and itself) are defined as zero. The neural dissimilarity matrix can be compared to the dissimilarity matrices posited by a number of models (in this example, model 1 and model 2). In the case of Bracci et al. (2015), the authors used a multiple regression (for each individual participant) using the model dissimilarity matrices as dependent variables and the neural dissimilarity matrices as independent variables. Differences between the computed regression coefficients were compared using pairwise $t$ tests to reveal which models best explained the neural dissimilarity. This resulted in bar graphs similar to that seen in this figure, which emphasize the degree to which each model can be related to the observed brain activity shown in the neural dissimilarity matrix, with beta estimating the explanatory contribution of each regressor (in this case, the two models).

Regardless of how the semantic model was generated, the clusters of organization within the LOTC and VOTC (effectors, noneffectors, faces) did indeed suggest an organization based on function. Tools (objects used as effectors) are represented close to body effectors in the OTC, and these regions are functionally connected to the left intraparietal sulcus (IPS) and left premotor cortex (Bracci et al., 2012). Interestingly, a whole-brain analysis run by Bracci et al. (2015) revealed additional clusters of frontal and parietal activation that were significantly and positively associated with the semantic similarity model once it was contrasted with the other four models. These areas were the left and right superior parietal lobule, left inferior parietal lobule, left ventral premotor cortex, and left dorsal premotor cortex. This may provide greater insight into the role of these areas in action perception and execution. Certainly, it supports the claim that the OTC is functionally connected to these regions. This association could be a reflection of the sensorimotor and functional similarity of body part representations seen in these areas (Rizzolatti and Craighero, 2004), but it may also highlight overlapping pathways relevant for semantic meaning and action processing. For example, the left IPS has been implicated both in the processing of words and objects (Devereux et al., 2013) and in the representation of observed action goals (Hamilton and Grafton, 2006)

Bracci et al. (2015) posited that semantic organization in the OTC could be a function of connectivity demands. Body part information related to action would feedforward to downstream motor areas, while areas representing faces would be strongly connected to regions involved in social cognition. These claims may be supported by new findings in studies on the movement disorder limb apraxia, which indicate that damage to the OTC often underlies defective pantomimed tool use, defective imitation of pantomimes, and defective imitation of meaningless gestures (Buxbaum et al., 2014; Hoeren et al., 2014). In addition, lesions to the OTC have been implicated in impaired (tool-related) action recognition (Tarhan et al., 2015). Such results confirm 
the idea that regions in the OTC could act as an upstream hub for multiple tasks reliant on body part recognition. Networks that include the OTC could have a semantic structure built in from the very beginning. Take action recognition as an example. Processing of the semantic aspects of action recognition is sometimes considered to rely on top-down cognition (Wurm et al., 2014; Davey et al., 2015). The results of Bracci et al. (2015) suggest that the way in which semantic and visual information are integrated for the purpose of action recognition may not rely primarily on top-down processing. If regions with semantic organization in the OTC and other areas (i.e., IPS, premotor cortex) are functionally linked, then the semantic nature of the observed stimulus could be derived from the pathway through which the stimulus information travels. Therefore, broad semantic information regarding the action context may in fact be integrated much earlier, following the observation of body parts. A recent study by Wurm and Lingnau (2015) appears to support this claim, but more work is needed to confirm the hypothesis.

Bracci et al. (2015) have increased our understanding of how body parts are represented in the OTC. They indicate that semantic information regarding the possible context in which body parts are observed may be available surprisingly early, during visual processing, due in part to the organization of visual processing regions. More importantly, their results have implications for how we discuss tasks reliant on body part recognition. Further study of the semantic organization in OTC could shed new light on processes as varied as action recognition and imitation, or even social cognition as a whole.

\section{References}

Belongie S, Malik J, Puzicha J (2002) Shape matching and object recognition using shape contexts. IEEE Trans Pattern Anal Mach Intell 24:509-522. CrossRef

Bracci S, Ietswaart M, Peelen MV, Cavina-Pratesi C (2010) Dissociable neural responses to hands and non-hand body parts in human left extrastriate visual cortex. J Neurophysiol 103: 3389-3397. CrossRef Medline

Bracci S, Cavina-Pratesi C, Ietswaart M, Caramazza A, Peelen MV (2012) Closely overlapping responses to tools and hands in left lateral occipitotemporal cortex. J Neurophysiol 107: 1443-1456. CrossRef Medline

Bracci S, Caramazza A, Peelen MV (2015) Representational similarity of body parts in human occipitotemporal cortex. J Neurosci 35: 12977-12985. CrossRef Medline

Buxbaum LJ, Shapiro AD, Coslett HB (2014) Critical brain regions for tool related and imitative actions: A componential analysis. Brain 137:1971-1985. CrossRef Medline

Davey J, Rueschemeyer SA, Costigan A, Murphy N, Krieger-Redwood K, Hallam G, Jefferies E (2015) Shared neural processes support semantic control and action understanding. Brain Lang 142:24-35. CrossRef Medline

Devereux BJ, Clarke A, Marouchos A, Tyler LK (2013) Representational similarity analysis reveals commonalities and differences in the semantic processing of words and objects. J Neurosci 33: 18906-18916. CrossRef Medline

Downing PE, Jiang Y, Shuman M, Kanwisher N (2001) A cortical area selective for visual pro- cessing of the human body. Science 293:24702473. CrossRef Medline

Grill-Spector K, Malach R (2004) The human visual cortex. Annu Rev Neurosci 27: 649-677. CrossRef Medline

Hamilton AF, Grafton ST (2006) Goal representation in human anterior intraparietal sulcus. J Neurosci 26:1133-1137. CrossRef Medline

Hoeren M, Kümmerer D, Bormann T, Beume L, Ludwig VM, Vry MS, Mader I, Rijntjes M, Kaller CP, Weiller C (2014) Neural bases of imitation and pantomime in acute stroke patients: distinct streams for praxis. Brain 137: 2796-2810. CrossRef Medline

Kriegeskorte N, Mur M, Bandettini P (2008) Representational similarity analysis-connecting the branches of systems neuroscience. Front Syst Neurosci 2:4. CrossRef Medline

Orlov T, Makin TR, Zohary E (2010) Topographic representation of the human body in the occipitotemporal cortex. Neuron 68:586600. CrossRef Medline

Rizzolatti G, Craighero L (2004) The mirrorneuron system. Annu Rev Neurosci 27:169192. CrossRef Medline

Tarhan LY, Watson CE, Buxbaum LJ (2015) Shared and distinct neuroanatomic regions critical for tool-related action production and recognition: evidence from 131 lefthemisphere stroke patients. J Cogn Neurosci 27:2491-2511. CrossRef Medline

Urgesi C, Berlucchi G, Aglioti SM (2004) Magnetic stimulation of extrastriate body area impairs visual processing of nonfacial body parts. Curr Biol 14:2130-2134. CrossRef Medline

Wurm MF, Lingnau A (2015) Decoding actions at different levels of abstraction. J Neurosci 35:7727-7735. CrossRef Medline

Wurm MF, Hrkać M, Morikawa Y, Schubotz RI (2014) Predicting goals in action episodes attenuates BOLD response in inferior frontal and occipitotemporal cortex. Behav Brain Res 274:108-117. CrossRef Medline 